\title{
Separation of salt and sweet waters in an area of former mines
}

\author{
P. P. Prochazka ${ }^{1} \&$ V. Dolezel ${ }^{2}$ \\ ${ }^{1}$ Czech Technical University in Prague, Czech Republic \\ ${ }^{2}$ Technical University Pardubice, Czech Republic
}

\begin{abstract}
In old deep abandoned mines, the problem of separation of the layers of a different nature needs to be solved. The problem discussed in this paper relates to mines situated near Prague, capital of the Czech Republic and is concerned with a separation of two kinds of underground water, one being salt (saline) and the other sweet (fresh) water. The layer of the sweet water is positioned at approximately $200 \mathrm{~m}$ below the surface, then there was originally a layer of watertight clay (hydrogeological insulator) and from some $300 \mathrm{~m}$ downwards, salt water occurs. A problem arose when the shafts, at a depth of $1000 \mathrm{~m}$, have been abandoned due to canceling the mine workings. During mining, pumps were used and no permeability was enabled. Since the mining has stopped, there is no reason to continue pumping and the danger of mixing both kinds of water has occurred. It was decided, in order not to damage the sweet water which is also used for wells and subsoil irrigation, that a stopper should be added into the clay layers to suppress any possibility of mixing both kinds of water. The problem then was oriented to answer several questions. One of the most important that was put forward was on the relationship between permeability coefficients and the thickness of the stopper. Another one was concerned with the optimization of expenses involving the required permeability, expressed in terms of economic indicators, the thickness depending on expenses, etc. As a numerical model has been prepared and it was necessary to feed it by reasonable input data, a scale model from physically equivalent materials was created and tested. The mutual influence of both models, mathematical and experimental, offered a very powerful tool for well-prepared reports on how the stopper should be constructed.

Keywords: scale modeling, boundary element method, leakage problem.
\end{abstract}




\section{Introduction}

The government of the Czech Republic decided that some deep mines would be closed because of their low efficiency. Their location, near the capital Prague underlined the decision, as the air and ground pollution problem should be improved in the capital. The only problem, which occurred before the mines were closed, consisted of the existence of two large layers of different kinds of water. The lower one contained salt and the upper sweet water. The salt water could not be allowed to pollute the upper layer, as plenty of wells in villages in the neighborhood of the mine could be contaminated. Two horizontal clay layers (insulant and semiinsulant) divide both large layers containing salt water and fresh water. Before establishing the deep mines, the vertical shafts were constructed with concrete linings. The salt water was draining away all the time during the existence of the mines. The drainage was designed very carefully to avoid possible infiltration of the salt water to the upper layer. Before the mines abandonment it was necessary to ensure the prevention of contact of both kinds of waters in the shafts. Several alternatives were put forward from which the most efficient appeared to be one which proposed filling up the shafts with spoil soil and the creation of a sealing stopper from soft clay at the horizons between the salt water and fresh water. The material for spoil soil will be delivered from deposits; their distance from the shafts is not too far. Such a material will be disparate; starting from grains of sand to big peaces of slate, slaty clay, and sandstone, reaching to nearly $1 \mathrm{~m}^{3}$. In order to recover the hydrogeological insulation between both kinds of water, in the depth of 220-300 m, slaty clay will be delivered and an insulating stopper will help keep the split of both kinds of water. A cross-section of the situation is depicted in Fig. 1.

The aim of this paper is to describe the design of the stoppers applied to splitting the contact horizon using coupled equations of effects of streaming water and deforming skeleton. The results from the laboratory are implemented to the numerical model in which optimal material properties of the stopper are sought. The radius of the shafts is $4.45 \mathrm{~m}$.

\section{Description of construction of the sealing stoppers}

After abandoning the mine and the horizontal seams and access horizontal shafts, only vertical shafts, which ended at the bottom of the horizontal seams, are of our interest. At this stage all technology equipment was removed and partly destroyed. The saline water started to increase its water level and after some time (c. 3 months, which was an equivalent of $300 \mathrm{~m}$ increase of the level of the salt water), a spoil material was tipped from the surface into the shaft. It was necessary to equilibrate the levels of the stewed spoil material and that of saline water in such a way that the dry strewed material does not outtop the water level. This stage lasted quite a long time, as the sedimentation of the spoil soil had to be ensured. The sedimentation was enforced by adding coagulators. In the same time the possibility of two or more stoppers were assessed. In the frame of this solution the permeability of stoppers was determined in dependence of their 
material properties. The coefficient of filtration $(\mathrm{m} / \mathrm{s})$ was gained from laboratory tests in a range of 2.5-4.0 $\left(* 10^{-11}\right)$. Classification of spoil material aimed to filling the shaft and classification of clay sealing material from the standpoint of geomechanical properties comes out from a description of specimens and from results from the laboratory and field (in situ) examinations. With regard to unexpected high groundwater affluent and the breakdown of the electrical power system control room and water-pumping station, water pumping became impossible; this is why backfilling into water is necessary. It is approved that so called dry rockfill can also invoke some technical difficulties resulting from technological approach.

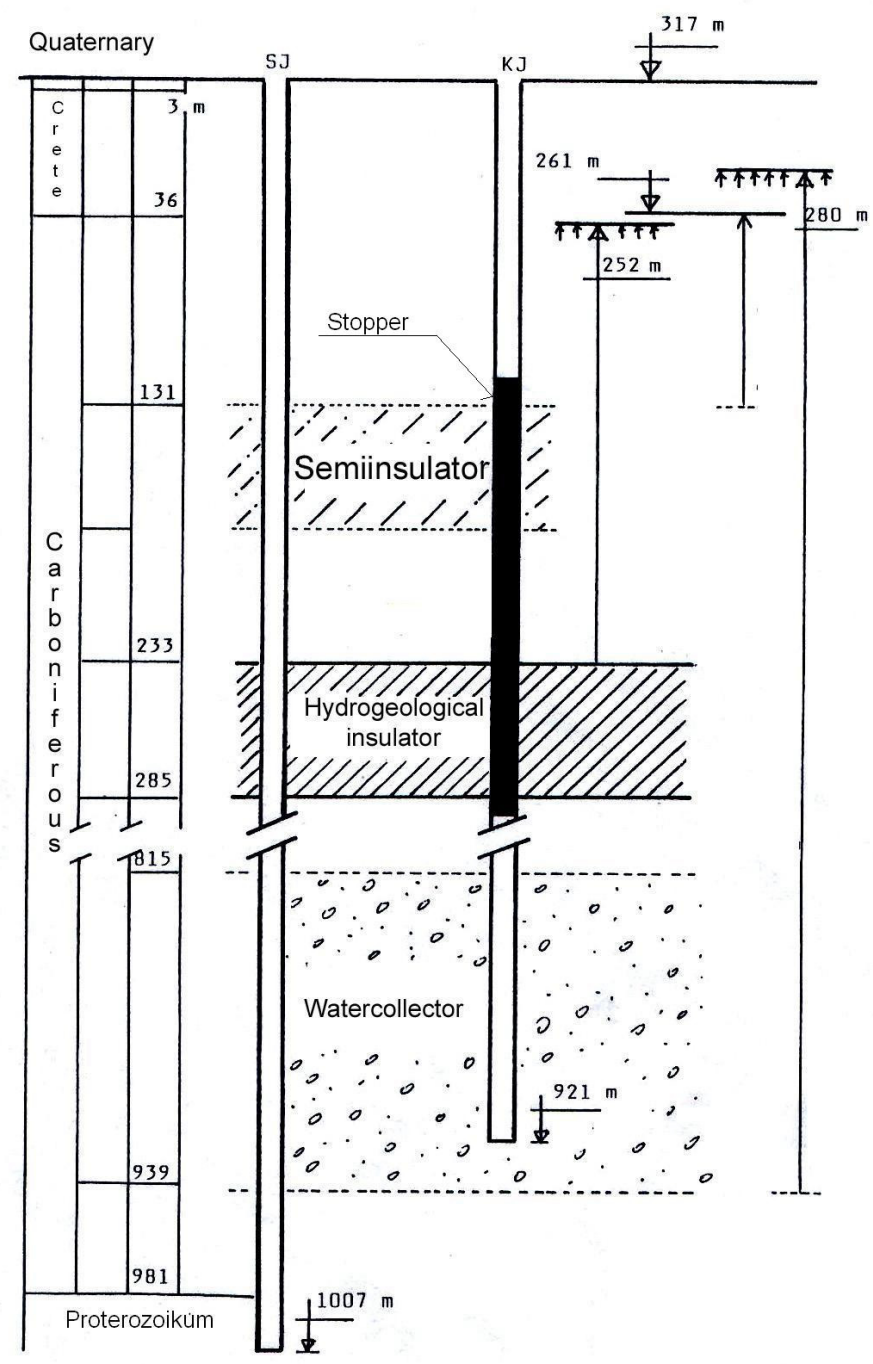

Figure 1: Cross-section of insulation by one sealing stopper. 
The principle of the whole problem consists in an assessment of timedependent behaviour of the backfill, which is found in the subsoil of sealing stoppers, especially concerning its sedimentation and compression. These factors basically influence the function of a sealing stopper.

Providing a decreasing inlet of water to the shafts in dependence of the increasing water level and a rate of strew of $20 \mathrm{~m}$ to $40 \mathrm{~m}$ a day it is possible to realize such a situation, when backfill strewing will be faster then the water level of the increasing water. Then such a situation can occur that at the height of the dry backfill higher than approximately $20 \mathrm{~m}$ due to larger value of angle of internal friction the principal part of the vertical load caused by volume weight is transferred to the lateral sides of the shafts. This phenomenon is dependent on moistening of this material. Correct function of the sealing stopper on such subsoil is very hard and difficult to ensure. If we take into consideration additional inflows of underground water, damage of the sides of shafts, or fluctuation of surface water, it could happen that sinking of the ground of the stoppers appears to be out of control and, consequently, undesirable free space and holes could be created. Too soft stoppers result in a decrease of its required sealing ability. In this case the backfill would be moistened and afterwards it attains the required settlement.

The next problem of identification of the material being necessary for sealing of the stoppers appears to be an assessment of the mechanically physical properties of a bulk spoil material in a high water column (almost to $600 \mathrm{~m}$ ). By virtue of the following analysis of mechanical and physical properties of the soil and rock in both shafts we may conclude: There is a high degradation of mechanical and deformational characteristics of materials due to the water. Reduction of the deformational characteristics (E - Young's modulus) from about 50 to $60 \%$ and mechanical properties from about 30 to $40 \%$ results from many performed tests, Tab. 1 , where $R_{d}$ is the compressive strength, $R_{t}$ is the tensile strength, $E_{o}$ is the deformation modulus of elasticity, $v$ is Poisson's number, $\phi$ is the angle of internal fiction, and $R_{\text {sh }}$ is the shear strength. To this it is necessary to count a decrease of the above values owing to transport, incidence and climatic influences, setting material into the shafts, etc. Because of a clash of the backfill material against the water surface and its subsequent spreading out the total decrease of its strength occurs and total destruction of the material appears.

Before placement the relationship between solid and lump material and cohesionless material is estimated at c 1:4. This ratio, after performing the backfill into the water, will further worsen the solid and cohesive material. Then, the relationship between solid and lump material and cohesionless material is changed to $\mathrm{c} 1: 6$.

In this technology it is almost impossible to make a selection of the quality of the spoil material. The next problem is to assess the material's properties and mechanical hydraulic behavior of the backfill.

In principle two assumptions exist about the behavior of the backfill below the water: 
1. The supporting skeleton of the backfill will have a relatively large porosity ( 30 to $40 \%$ ). Spaces between single solid pieces will successively be filled with fine sediment elements. However, there is a possibility that in the liquid of relatively high density (to $1.3 \mathrm{~g} / \mathrm{cm}$ ) clay contacts are created between single grains of the skeleton, which tighten individual cavities and therefore prevent the next settling.

Table 1: $\quad$ Material properties of the spoil soil.

\begin{tabular}{|l|l|l|l|l|l|l|l|l|}
\hline & \multicolumn{2}{|c|}{$\begin{array}{c}\text { middlegr. } \\
\text { sandstone }\end{array}$} & \multicolumn{2}{c|}{$\begin{array}{c}\text { sandy } \\
\text { siltstone }\end{array}$} & \multicolumn{2}{c|}{$\begin{array}{c}\text { silt } \\
\text { sandstone }\end{array}$} & \multicolumn{2}{c|}{$\begin{array}{c}\text { clay } \\
\text { siltstone }\end{array}$} \\
\hline & shaft & dump & \multicolumn{1}{|c|}{ shaft } & dump & shaft & dump & shaft & dump \\
\hline \hline $\mathrm{R}_{\mathrm{d}}$ & 46.9 & 35.2 & 78.3 & 15.1 & 67.6 & 66.1 & 15.1 & 15.1 \\
\hline $\mathrm{R}_{\mathrm{tp}}$ & 2.47 & & 2.98 & & & & 2.9 & \\
\hline $\mathrm{E}$ & 7487 & & 10267 & 2550 & 18063 & 6280 & 9528 & \\
\hline $\mathrm{E}_{\mathrm{o}}$ & 6603 & 1200 & 10209 & & 14816 & & 7366 & \\
\hline$v$ & 0.19 & & 0.26 & & 0.36 & & 0.4 & \\
\hline $\mathrm{R}_{\mathrm{sh}}$ & 4.9 & & 6.7 & & 9.9 & & 7.55 & \\
\hline$\phi$ & 61.6 & 28 & 65.2 & 31.2 & 60.2 & & 67.9 & \\
\hline
\end{tabular}

2. If the backfill consist mainly of the clay material without solid grains, then single parts of clay will be destructed into single clay elements and subsequent sedimentation can appear. Therefore a new compact clay layer has to be created. The same process will obviously proceed also during filling the clay material of the sealing stopper into the shaft. However, in the same time, the consolidation can be slowed down remarkably owing to the upward pressure and the pore pressures. However, the above-mentioned process can hardly be quantified. In any case it is necessary to support this process with the addition of coagulators.

Except for porosity of virgin material in the range $25-45 \%$ the loose material possesses also great external void fraction (ratio of the volume lacunae between single pieces to general volume of the loose material). In this phase pieces are wedged, spun and crushed until a certain value of the load, the fresh loose material is very permeable. Therefore, it is also very compressible. The speed of the sedimentation is, in this start-up phase, more dependent on changes of the moisture than on time. Only after minimizing of the void fraction, which can take place with depths greater than $50 \mathrm{~m}$, can the process of the classical filtration consolidation with an appropriate reduction of the porosity obviously commence. This process is already markedly time-dependent. The consolidation successively slows at the stable level of the normal load. From results of the shear tests it follows that the values of the shear strength of the clay loose material are impossible to deduce from shear strengths given from entire specimens. It can be stated that the more coherently is overburden removing soil, the less cohesive is its loose material.

Shear tests with loose materials under normal pressures to the $3 \mathrm{MPa}$ brought about piece of knowledge that overstepping the normal tension by about 1-1.5 
MPa causes crashing of their structure. This process is in compliance with geostatic pressure in depths $50-75 \mathrm{~m}$. At the same time the angle of internal friction decreases by one half and the growth of the cohesion is doubled.

This phenomenon is observed at the clay loose material with moisture of max. $25-30 \%$ (in dry matter). If the moisture is higher, the described phenomenon shifts to the area of the lower pressure, in some case it disappears. In order to gain this change it is sufficient to moisten the specimens about 5-7\%. Difficulties of the laboratory determination of the shears strength of clay loose material results from the high normal stress. At small stress states the loose material is softened, the total parameters of the shear strength are basically coincident with effective parameters. In the next increasing of the normal stress over certain limit (1-1.5 MPa) pore pressure and air pressure gradually increase. After total decreasing of the void fraction in the loose material the normal stress due to increase of loading is transmitted only to water. Mobilized shear stress in the loose material does not virtually increase. The settlement of the surface of loose material is an external manifestation of various deformation processes that proceed in the entire body. Changes are time-dependent, their size and speed depend on the possibility of the saturation of water. Lumps of the freshly based loose clay material to the dry environ have a solid to rigid consistence. In consequence of the unloading at the excavation of the sealing clay material is in pores of single pieces and the negative pore pressure, because lumps easily absorb the moisture from the neighborhood.

The loose clay material with the initially considerable porosity $(40 \%$, for example) after some time at higher normal pressure (cca 1.5 MPa) turns to become waterproof and airtight.

As far as the deformation processes pass under restricted access of the waters they are caused by crushing of lumps on contacts of the surfaces. After the forthcoming reduction of the void fraction a closure of the air occurs. In this way the agglutinate material is almost waterproof. For such materials, it threads no extreme worsening of strength and deformation characteristics.

On the contrary, the worst properties of the loose material are attained if all space is fulfilled by water. External influences of water are the most dangerous for dry loose material, as the loose material, in the beginning, is permeable. In this case we explain, for instance, of the turn of the loose material to the dump. Gradual plasticization will lead to homogenization of the soil. Mostly, we only obtain the soft till pulpy (pasty) consistence.

Characteristics changed in this way do not allow the next rising of the thickness of the outer dump. At the moment of the placing of next thicknesses all addition of the normal load relates to the pore water with the zero shear strength. The dump becomes unstable as a consequence of active forces under current stagnation of passive forces. The settlement of the soft till mash material can be smaller, providing prevention of the extrusion of the dump to the sides. This case is also possible at the pelted shaft of the deep mine.

Now we try to make an analysis of the progress at which the sealing clay material stopper spill from the big high to the water. The main problem is the behavior of the diffused clay material in the surrounding water. The 
gravitational effect dominates during the settlement of grains. The interaction between distinctive grains begins at that time, when forces among single elements dominate over gravitational forces.

\section{Numerical description of streaming water}

In this section we briefly formulate well known relations with the only target that some known coefficients will have special meaning, and are introduced for tuning with results from scale models. After that, simulation and optimization of the different types of stoppers and their number are considered in numerical models. We start with denotation of two phases composite. The water pressure will be described by function $p=p(\boldsymbol{x}, t)$ depending on a position and time. Displacement field in the skeleton is described by function $\boldsymbol{u}(\boldsymbol{x}, t)=\left\{u_{1}(\boldsymbol{x}, t), u_{2}(\boldsymbol{x}, t), u_{3}(\boldsymbol{x}, t)\right\}$. Time dependent changes of pores are denoted as $\dot{\Theta}$. Total stress is split into effective stress and the influence of water pressure:

$$
\sigma_{i j}=\sigma_{i j}^{\mathrm{eff}}-p \delta_{i j} .
$$

where $\delta_{i j}$ is Kronecker's delta indicating that the water pressure does not effect the shear components of stress tensor. The same is valid for strains. If we write the tensor of stresses artificially in terms of vector:

$$
\sigma=\left\{\sigma_{x x}, \sigma_{y y}, \sigma_{z z}, \sigma_{x y}, \sigma_{x z}, \sigma_{y z}\right\}^{\mathrm{T}} .
$$

We also use this notation for strain tensors:

$$
\varepsilon=\left\{\varepsilon_{x x}, \varepsilon_{y y}, \varepsilon_{z z}, \varepsilon_{x y}, \varepsilon_{x z}, \varepsilon_{y z}\right\}^{\mathrm{T}} .
$$

Then

$$
\sigma=\sigma^{\text {eff }}-p \boldsymbol{m}
$$

where $p$ is scalar and $\boldsymbol{m}=\{1,1,1,0,0,0\}^{\mathrm{T}}$. The effective stress can be expressed as:

$$
\dot{\sigma}^{\mathrm{eff}}=\boldsymbol{D}\left(\dot{\boldsymbol{\varepsilon}}+\dot{\boldsymbol{\varepsilon}}^{\mathrm{pl}}-\dot{\boldsymbol{\varepsilon}}_{\mathrm{p}}\right)
$$

where dot means time derivative, $\boldsymbol{D}$ is stiffness matrix of elastic media, $\varepsilon$ is elastic stress tensor, $\varepsilon^{\mathrm{pl}}$ is the strain in plastic domain, and $\varepsilon_{\mathrm{p}}$ means the strain tensor caused by water pressure. The time increment of the latter strain (velocity of deformation of the basic material due to pore pressure) can be expressed as:

$$
\dot{\varepsilon}_{p}=-\boldsymbol{m}\left(\frac{\dot{p}}{3 K_{z}}\right),
$$


where $K_{z}$ is the bulk modulus of the basic material. Compressing the liquid using the pore pressure we get after easy algebra the following relation:

$$
\dot{\sigma}=\dot{\sigma}^{e f f}-\dot{p} \boldsymbol{m}=\left(\dot{\boldsymbol{\varepsilon}}+\dot{\boldsymbol{\varepsilon}}^{\mathrm{pl}}\right)-\alpha \boldsymbol{m} \dot{p}
$$

where $\alpha=1-\frac{1}{3 K_{z}}, K_{z}$ is the coefficient of expansion due to the water. The time increment of changes of pores can be expressed as

$$
\dot{\theta}=\alpha \boldsymbol{m}^{\mathrm{T}} \dot{\boldsymbol{\varepsilon}}+\left[\frac{n}{K_{k}}+\frac{1-n}{K_{z}}+(1-\alpha) \frac{1}{K_{z}}\right] \dot{p}
$$

where $n$ (porosity) is the increment of the volume of skeleton due to an increment of pore pressure. On the other hand Darcy's law is valid

$$
n \operatorname{div} \mathbf{v}=-\dot{\theta}
$$

where $\boldsymbol{v}$ is the vector of relative velocity of the displacement of the liquid with respect to the displacement of the skeleton. According to Darcy's law the relative velocity of streaming liquid is proportional to the gradient of pore pressure:

$$
\begin{gathered}
-\frac{c}{K_{s}} \Delta p+\lambda \dot{p}+\alpha \boldsymbol{m}^{\mathrm{T}} \dot{\boldsymbol{\varepsilon}}=0, \lambda=\frac{n}{K_{k}}+\frac{1-n}{K_{z}}-(1-\alpha) \frac{1}{K_{z}}, \\
\text { for } \alpha \approx 0:-\frac{c}{K_{s}} \Delta p+\alpha \boldsymbol{m}^{\mathrm{T}} \dot{\boldsymbol{\varepsilon}}=0,
\end{gathered}
$$

where $K_{s}$ is the bulk modulus of elasticity of the skeleton, $c=\frac{K_{s} k}{\rho_{k} g}, \rho_{k}$ is the density of the fluid and $g$ is gravitation, $k$ is the coefficient of filtration.

The last equation together with the Navier equations creates a system of partial differential equations, which are solved by finite or boundary elements.

\section{Laboratory modeling}

The mathematical model serves for solving water pressures in pores and overall (total) stresses in the insulator. Note that the components of stress tensor are virtually impossible to determine in the experimental and "in situ" measurements. On the other hand, no numerical model can be applied without appropriate material and internal parameters. This is why experimental scale models were prepared and the process of construction of the insulator was 
simulated in the stands, which are partly glassed basins boxes. The sides of the stands are about $1.5 \times 1.5 \times 1.5 \mathrm{~m}^{2}$. Natural materials fill the stands and the water is pushed through the pores of the material.

Classification of the insulator material originates from the description of samples from the site and laboratory tests. With respect to unexpected inflow of ground water and to spare the energy, the continuation or drainage was excluded and the project expected no penetration of saline water to the upper level. Since the problem consists of the time dependent behavior of the insulator, particularly its sedimentation, compression, seepage, change of coefficients of filtration $\left(k_{r}=\right.$ $k_{\varphi}=0.8 k_{z}$; all these coefficients (radial $r$ and hoop $\varphi$ ) are dependent on $z$ direction, which is axial - vertical - coordinate), and the solution is strongly nonlinear. All these factors essentially influence the insulation function of the water stopper. Under assumption of decreasing inflow of water into the shafts in dependence of increasing level of water and the velocity of construction of the stopper (20 to $40 \mathrm{~m}$ a day) it is realistic to imagine a situation, the upper level of the stopper in which "outruns" the increasing water level. If the stopper is finished and the fresh water is let to fang to the shaft, both types of waters should not be in contact, i.e., the upper level of saline water and the lower level of the fresh water may not meet at any stage of construction.

Table 2: $\quad$ Water pressure at different points of the stopper.

\begin{tabular}{|c|c|c|c|c|c|c|c|c|}
\hline$z \mid r$ & 0.0 & 0.6 & 1.2 & 1.8 & 2.4 & 3.2 & 3.8 & 4.45 \\
\hline 40 & 0.000 & 0.000 & 0.000 & 0.000 & 0.000 & 0.000 & 0.000 & 0.000 \\
\hline 37.5 & 0.000 & 0.000 & 0.000 & 0.000 & 0.000 & 0.000 & 0.000 & 0.001 \\
\hline 35 & 0.000 & 0.000 & 0.001 & 0.002 & 0.002 & 0.001 & 0.001 & 0.002 \\
\hline 32.5 & 0.002 & 0.002 & 0.002 & 0.004 & 0.004 & 0.004 & 0.005 & 0.005 \\
\hline 30 & 0.004 & 0.007 & 0.011 & 0.013 & 0.015 & 0.015 & 0.017 & 0.019 \\
\hline 27.5 & 0.014 & 0.021 & 0.034 & 0.046 & 0.054 & 0.057 & 0.057 & 0.057 \\
\hline 25 & 0.037 & 0.060 & 0.107 & 0.148 & 0.175 & 0.189 & 0.194 & 0.195 \\
\hline 22.5 & 0.080 & 0.121 & 0.205 & 0.279 & 0.329 & 0.354 & 0.365 & 0.369 \\
\hline 20 & 0.150 & 0.223 & 0.378 & 0.518 & 0.609 & 0.658 & 0.673 & 0.675 \\
\hline 17.5 & 0.271 & 0.406 & 0.690 & 0.950 & 1.122 & 1.208 & 1.236 & 1.239 \\
\hline 15 & 0.483 & 0.725 & 1.248 & 1.733 & 2.056 & 2.221 & 2.266 & 2.265 \\
\hline 12.5 & 0.835 & 1.262 & 2.212 & 3.127 & 3.758 & 4.083 & 4.159 & 4.148 \\
\hline 10 & 1.358 & 2.084 & 3.759 & 5.496 & 6.801 & 7.518 & 7.684 & 7.650 \\
\hline 7.5 & 2.004 & 3.131 & 5.877 & 9.062 & 11.908 & 13.837 & 14.461 & 14.438 \\
\hline 5 & 2.542 & 4.050 & 7.931 & 13.020 & 18.778 & 24.742 & 28.426 & 29.187 \\
\hline 2.5 & 2.597 & 4.205 & 8.440 & 14.416 & 22.530 & 35.209 & 58.596 & 74.068 \\
\hline
\end{tabular}

The scale modeling proves that the correct function of the stopper is very tough and difficult. Moreover, the scale modeling proved again that in the final stage of material characteristics changed principally, e.g., Young's modulus E dropped by 50 to 60 percent and toughness by 30 to 40 percent. 


\section{Conclusions}

In this paper coupled modeling (sort of back analysis) is applied to solution of optimal design of sealing stoppers, which should substitute natural clay insulators dividing two sorts of water. They are built up as recovering of the situation after abandonment of deep mines. The numerical model serves as a tool for decisions on how to design the stoppers in an optimal way, the scale models are tools for delivering necessary data to the numerical model.

We first introduce very important distribution of coefficients of filtration $k$ $(\mathrm{m} / \mathrm{s})$ in vertical direction depending on moisture $w(\%)$, which was derived from large scale of scale models. The relation is depicted in Fig. 2.

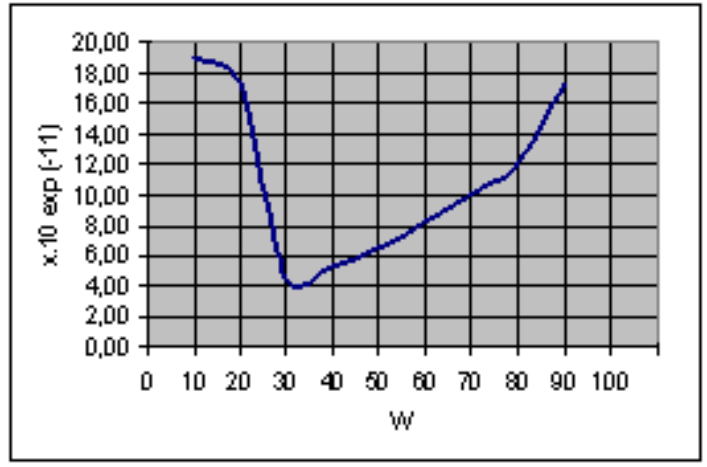

Figure 2: Relation of coefficient of filtration and moisture.

From finite elements distribution of water flow is computed and similarly from Lame's equations stresses are calculated using finite elements again. In certain points strength in the sense of Mohr-Coulomb hypotheses (ideally elastoplastic law) is attained and the disturbance function desires change in its value at such points (having impact on the coefficients of filtration and others). The resulting pressure for optimal configuration of the stopper appears in Tab. 1, where rows denote the distance from the bottom of stopper in $\mathrm{m}, r$ is the radius measured in meters from the shaft axis. It is obviously seen that the highest values of pressure are observed at the outer ring of the stopper and on the lower boundary. Moreover, $40 \mathrm{~m}$ from the lower boundary almost no pressure is obtained in the final stage of the construction of the stopper. The distribution of water pressure along the lower boundary shows that the higher pressure is observed at the concrete lining, the lowest at the axis.

\section{Acknowledgments}

The first author was financially supported by GA AV ČR, project No. IAA 2119402, the second author appreciates the financial support of GA ČR No. $103 / 05 / 0679$. 